\title{
Temperature dependent resistance of magnetic tunnel junctions as a quality proof of the barrier
}

\author{
U. Rüdiger, ${ }^{\text {a) }}$ R. Calarco, U. May, K. Samm, J. Hauch, H. Kittur, M. Sperlich, \\ and G. Güntherodt \\ II. Phys. Institut, RWTH Aachen, 52056 Aachen, Germany
}

\begin{abstract}
Tunnel junctions of $\mathrm{Co}(10 \mathrm{~nm}) / \mathrm{AlO}_{x}$ (nominally $\left.2 \mathrm{~nm}\right) / \mathrm{Co}(20 \mathrm{~nm}$ ) have been prepared by molecular beam epitaxy applying a shadow mask technique in conjunction with an UV light-assisted oxidation process of the $\mathrm{AlO}_{x}$ barrier. The quality of the $\mathrm{AlO}_{x}$ barrier has been proven by x-ray photoelectron spectroscopy and temperature dependent tunneling magnetoresistance (TMR) measurements. Optimum-oxidized tunnel junctions show a TMR of $20 \%$ at $285 \mathrm{~K}$ and up to $36 \%$ at $100 \mathrm{~K}$. At 285 $\mathrm{K}$ the TMR values as a function of oxidation time are not symmetric about the optimum time. For underoxidized junctions the TMR is reduced more strongly than for overoxidized junctions. The temperature dependence of the junction's resistance is a clear and reliable indicator whether pinholes (or imperfections) contribute to the conduction across the barrier.
\end{abstract}

Magnetic tunnel junctions (MTJs) consisting of two ferromagnetic electrodes separated by a thin insulating layer (typical $\mathrm{AlO}_{x}$ ) show large tunnel magnetoresistance (TMR) at room temperature making them promising candidates for magnetic random access memory (MRAM) devices. ${ }^{1-3}$ Strong efforts have been spent on reducing the resistance $\times$ area $(R \times A)$ product by reducing the thickness of the typically used $\mathrm{AlO}_{x}$ insulating barrier $(<10 \AA)$. But not only the thickness of the insulating barrier, but also the oxidation process itself has a significant influence on the $R$ $\times A$ product. ${ }^{4}$ Different oxidation processes have been investigated and optimized in order to evaluate the efficiency and reliability for large scale oxidation of the barrier material. ${ }^{5-9}$ In comparison with other oxidation processes ultraviolet (UV) light-assisted oxidation ${ }^{10,11}$ generally leads to a factor of 10-100 lower $R \times A$ products and a significantly smaller bias dependence of the TMR for optimum oxidized samples. ${ }^{4}$ On the other hand, lowering the $R \times A$ product by minimizing the $\mathrm{AlO}_{x}$ barrier thickness may enhances the probability of a pinhole (or direct metal-metal contact) formation ${ }^{12}$ in the insulating barrier. The recent observation of large MR effects in magnetic nanocontacts in the presence of trapped domain walls ${ }^{13-15}$ suggests that spin-dependent domain wall scattering ${ }^{16-18}$ can significantly contribute to the MR of MTJ. In order to distinguish spin-dependent tunneling conductance from conductance through pinholes the "Rowell criteria" can be applied. ${ }^{19}$ As recently discussed only the analysis of the temperature dependent resistance (conductance) seems to be reliable and gives an "easy-to-handle" control of the conduction mechanisms in MTJs. ${ }^{20,21}$

In this article we discuss the optimization of the UV light-assisted oxidation process of $\mathrm{AlO}_{x}$ barriers of $\mathrm{MBE}$ prepared $\mathrm{Co}(10 \mathrm{~nm}) / \mathrm{AlO}_{x}(2 \mathrm{~nm}) / \mathrm{Co}(20 \mathrm{~nm}) / \mathrm{Si}(100)$ junctions by controlling the oxidation process by $\mathrm{x}$-ray photo-

a) Author to whom correspondence should be addressed; electronic mail: ruediger@physik.rwth-aachen.de electron spectroscopy (XPS), TMR, and the temperature dependent $R \times A$ product as a function of oxidation time.

MTJs have been fabricated by shadow mask deposition using electron beam evaporation in UHV (base pressure $p$ $\left.=8 \times 10^{-11} \mathrm{mbar}\right)$. The area of the bottom and top Co electrodes separated by the $\mathrm{AlO}_{x}$ barrier is $(150 \times 150) \mu \mathrm{m}^{2}$. The 2 -nm thick Al layers have been deposited by $e$-beam evaporation followed by an in situ oxidation process in an $\mathrm{O}_{2}$ atmosphere using a $15 \mathrm{~W}$ UV lamp inside the chamber. For XPS measurements the monochromatized $\mathrm{Mg} K_{\alpha}$ emission line of $E=h \nu=1253.6 \mathrm{eV}$ has been employed. Low bias dc resistance and TMR measurements have been performed in a variable temperature high magnetic-field cryostat.

Figure 1 shows the XPS spectra of UHV prepared $\mathrm{Al}(2$ $\mathrm{nm}) / \mathrm{Co}(20 \mathrm{~nm})$ double layers in the $\mathrm{Al} 2 s$ and $2 p$ core level region $(55-135 \mathrm{eV})$. A nonoxidized $\mathrm{Al}(2 \mathrm{~nm}) / \mathrm{Co}(20 \mathrm{~nm})$ double layer acts as reference for the XPS investigation of

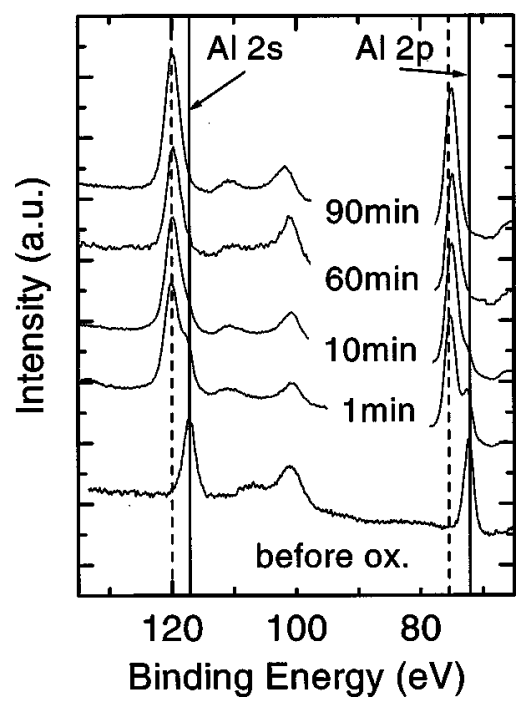

FIG. 1. XPS spectra of the $\mathrm{Al} 2 s$ and $2 p$ core levels of $\mathrm{Al}(2 \mathrm{~nm}) / \mathrm{Co}(20 \mathrm{~nm})$ double layers as function of oxidation time $t_{\mathrm{ox}}$. 


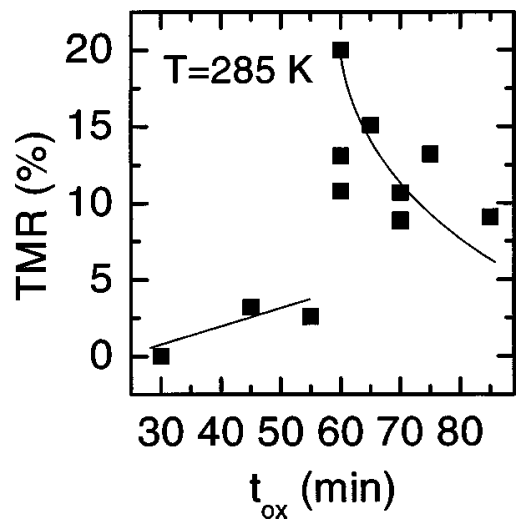

FIG. 2. Tunneling magnetoresistance of $\mathrm{Co}(10 \mathrm{~nm}) / \mathrm{AlO}_{x}(2 \mathrm{~nm}) / \mathrm{Co}(20 \mathrm{~nm})$ junctions as function of oxidation time at $T=285 \mathrm{~K}$.

the oxidation process. Already after 1 min of UV lightassisted oxidation the $\mathrm{Al} 2 s(117.2 \mathrm{eV})$ and $2 p(72.2 \mathrm{eV})$ peaks are clearly shifted by approximately $2.1 \mathrm{eV}$. The presence of a double peak structure for both levels indicates that the Al layer is not fully oxidized. Nevertheless, most of the $\mathrm{Al}$ has been oxidized within the first $10 \mathrm{~min}$. After $60 \mathrm{~min}$ of oxidation the double-peak like structure of the $\mathrm{Al}$ core levels disappears, indicating a fully oxidized Al layer. For oxidation times larger than $60 \mathrm{~min}$ the XPS spectra in the $\mathrm{Al}$ core level region do not change anymore. XPS Co core level $\left(2 p_{1 / 2}\right.$ and $\left.2 p_{3 / 2}\right)$ spectra also support that 60 min oxidation time is the optimum oxidation time achieving a fully oxidized $\mathrm{Al}$ layer without oxidizing the underlying $\mathrm{Co}$ electrode. $^{22}$

For junctions oxidized during $t_{\mathrm{ox}}=60 \mathrm{~min}$ the TMR at $T=100 \mathrm{~K}$ exhibits a sharp maximum of $36 \%$. Slightly underand overoxidized samples (45 and 75 min oxidation time) reveal a reduction of the TMR to approximately $20 \%$ symmetric about $t_{\mathrm{ox}}=60 \mathrm{~min}$.

Figure 2 shows TMR values of $\mathrm{Co}(10 \mathrm{~nm}) / \mathrm{AlO}_{x}(2 \mathrm{~nm}) /$ $\mathrm{Co}(20 \mathrm{~nm})$ junctions as function of oxidation time at $T$ $=285 \mathrm{~K}$ with a maximum TMR for $t_{\mathrm{ox}}=60 \mathrm{~min}$ of approximately $20 \%$. The $R \times A$ product for the optimum-oxidized sample is $160 \pm 60 \mathrm{k} \Omega \mu \mathrm{m}^{2}$. In contrast, the TMR values as a function of oxidation time are not symmetric about the optimum time. For underoxidized samples $\left(t_{\mathrm{ox}} \leqslant 55 \mathrm{~min}\right)$ the TMR is reduced more strongly than for the over-oxidized samples $\left(t_{\mathrm{ox}} \geqslant 65 \mathrm{~min}\right)$. For example the TMR for junctions with $t_{\mathrm{ox}}=55 \mathrm{~min}$ has been determined to be $2.5 \%$, whereas the TMR for a sample with $t_{\mathrm{ox}}=65 \mathrm{~min}$ is still $15 \%$. Also modestly overoxidized samples $\left(t_{\mathrm{ox}}=85 \mathrm{~min}\right)$ can show a TMR of approximately $10 \% .^{22}$

The observed strong decrease of the TMR of underoxidized samples as function of temperature can have different origins. For an interpretation one has to distinguish between the temperature dependence of the spin-dependent and spinindependent contribution to the resistivity. The decrease of the spin-dependent tunneling contribution with increasing temperature is based on the reduction of the magnetization (spin polarization) of the electrodes (especially at the electrode/barrier interface) due to the excitation of magnons and due to broadening of the Fermi distribution. ${ }^{23}$ For the

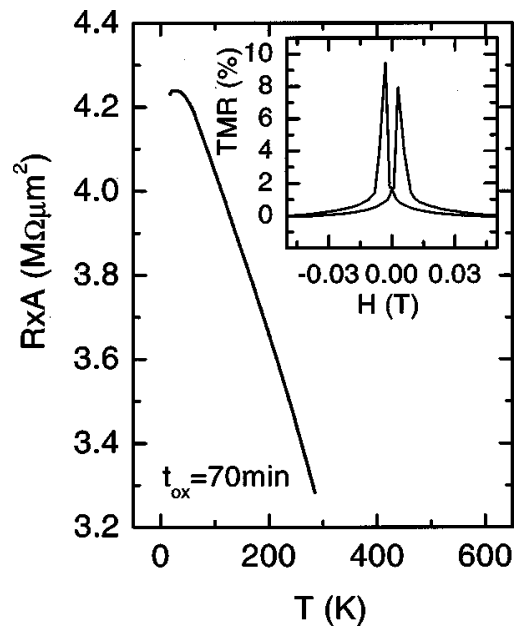

FIG. 3. The resistance $\times$ area $(R \times A)$ product of an overoxidized $\left(t_{\mathrm{ox}}\right.$ $=70 \mathrm{~min}) \mathrm{Co}(10 \mathrm{~nm}) / \mathrm{AlO}_{x}(2 \mathrm{~nm}) / \mathrm{Co}(20 \mathrm{~nm})$ junction exhibits an insulator-like temperature dependence. The inset shows a typical field dependent tunneling magnetoresistance curve with maxima for antiparallely magnetized electrodes (at $T=285 \mathrm{~K}$ ).

spin-independent contribution to the resistance a hopping process via localized states due to imperfections in the insulating barrier has been suggested. ${ }^{23}$ Such hopping processes can give rise to a temperature dependent reduction of the TMR much faster than based on magnon excitations only.

In a simple model underoxidation leads to a very thin $\mathrm{Al}$ interlayer between the $\mathrm{AlO}_{x}$ and the Co bottom electrode or to $\mathrm{Al}$ inclusions within the already formed $\mathrm{AlO}_{x}$ barrier. In the presence of a nonuniformly oxidized barrier $\left(\mathrm{Al} / \mathrm{AlO}_{x}\right.$ double layer) or nonoxidized $\mathrm{Al}$ inclusions within the oxide barrier the temperature dependence of a spin-independent tunneling contribution can be larger than in the case of an overoxidized sample with an additional $\mathrm{CoO}_{x}$ layer separating the Co bottom electrode and the $\mathrm{AlO}_{x}$ barrier. ${ }^{22}$

Additionally, a remaining $\mathrm{Al}$ layer on top of the Co bottom electrode can significantly reduce the surface spin polarization of the Co interface layer close to the Fermi energy $E_{F}$. Spin-polarized band structure calculations have shown that an $\mathrm{Al}$ termination of the Co bottom electrode strongly reduces the spin polarization of $\mathrm{Co}$ at the interface. ${ }^{24,25}$ Also randomly distributed $\mathrm{Al}$ inclusions can have an influence on the spin polarization of the tunneling current. With an increasing impurity concentration within the barrier the spin polarization of the tunneling current decreases, leading to a reduction of the TMR. ${ }^{26,27}$ Therefore, underoxidation can affect the performance of junctions more seriously than overoxidation. But it is still open why a similar asymmetric reduction of TMR at $T=100 \mathrm{~K}$ for underoxidized samples has not been observed.

For interpreting the temperature dependence of the TMR data one has to keep in mind that there is no strict evidence of a full antiparallel alignment of the two Co electrodes. Different temperature dependent coercivities of the electrodes may induce different degrees of antiparallelism as a function of temperature, therefore leading to the observed asymmetric reduction of TMR at $T=285 \mathrm{~K}$.

Figure 3 shows the $R \times A$ product as a function of tem- 


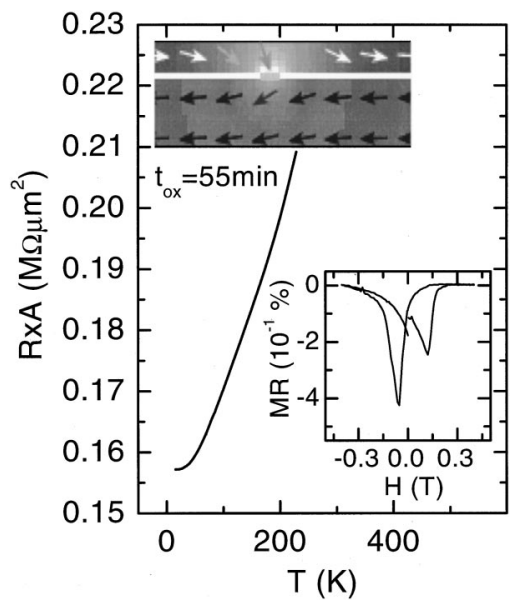

FIG. 4. The resistance $\times$ area $(R \times A)$ product of a slightly underoxidized $\left(t_{\mathrm{ox}}=55 \mathrm{~min}\right) \mathrm{Co}(10 \mathrm{~nm}) / \mathrm{AlO}_{x}(2 \mathrm{~nm}) / \mathrm{Co}(20 \mathrm{~nm})$ junction exhibits a metallike temperature dependence. The bottom inset shows an unusual field dependent magnetoresistance curve, which has minima for antiparallely magnetized Co electrodes (at $T=10 \mathrm{~K}$ ). The top inset gives the magnetization distribution of the two Co electrodes (10 and $20 \mathrm{~nm}$ thick) separated by a 2-nm-thick insulator in the presence of a pinhole.

perature for an overoxidized junction $\left(t_{\mathrm{ox}}=70 \mathrm{~min}\right)$ which decreases with increasing temperature as is typical for tunnel junctions. At $T=285 \mathrm{~K}$ the $R \times A$ product is approximately $3 \mathrm{M} \Omega \mu \mathrm{m}^{2}$. The inset of Fig. 3 shows a typical field dependent TMR curve with maxima for antiparallely magnetized electrodes.

In contrast, the $R \times A$ product of a sample showing no TMR $\left(t_{\mathrm{ox}}=55 \mathrm{~min}\right)$ increases with increasing temperature (see Fig. 4) pointing to metallic channels (pinholes) through the insulating barrier. ${ }^{20}$ In contrast to the TMR curve in the inset of Fig. 3 the MR curve of this sample shows minima for antiparallely magnetized electrodes (see bottom inset of Fig. 4). Generally, all investigated MTJ with a metal-like temperature dependent resistance also exhibit comparable MR curves. These unusual TMR curves can be interpreted on the basis of anisotropic MR, which has its origin in spinorbit coupling depending on the relative orientation of the current $\mathbf{I}$ and the magnetization $\mathbf{M} .^{28}$ Generally, in ferromagnets the resistance for $\mathbf{I} \| \mathbf{M}$ is larger than for $\amalg \mathbf{M}\left(R_{\|}\right.$ $\left.>R_{\perp}\right) .{ }^{28}$ The magnetization distribution during the magnetization reversal of the two Co electrodes (10 and $20 \mathrm{~nm}$ thick) separated by a 2-nm-thick insulator layer has been calculated in the presence of a 5-nm-wide pinhole and is shown in the top inset in Fig. 4 for antiparallely magnetized electrodes. ${ }^{29}$ The local magnetization distribution is indicated by arrows. Due to the presence of the pinhole there is a significant perpendicular magnetization component, which probably leads to the observed reduced resistance for antiparallely magnetized electrodes.

In summary, an UV light-assisted oxidation process of $\mathrm{Co}(10 \mathrm{~nm}) / \mathrm{AlO}_{x}(2 \mathrm{~nm}) / \mathrm{Co}(20 \mathrm{~nm})$ junctions has been investigated as a function of oxidation time. The TMR shows a sharp maximum as a function of oxidation time near $t_{\mathrm{ox}}$ $=60 \mathrm{~min}$, but the decrease of TMR for overoxidized junctions with increasing oxidation time is less pronounced than for underoxidized junctions. For an "easy-to-control" fabrication process of tunneling junctions a slight overoxidation seems to be favorable. Temperature dependent resistance measurements are a reliable method ruling out MTJ with pinholes (direct metal-metal point contacts).

This work was supported by the German Federal Ministery for Education and Research "BMBF" under Grant No. FKZ 13N7329 and the EC TMR Program "Submicron Magnetic Structures and Magneto-Transport Devices" (SUBMAGDEV).

${ }^{1}$ S. S. P. Parkin et al., J. Appl. Phys. 85, 5828 (1999).

${ }^{2}$ J. S. Moodera, L. R. Kinder, T. M. Wong, and R. Meservey, Phys. Rev. Lett. 74, 3273 (1995).

${ }^{3}$ W. J. Gallagher et al., J. Appl. Phys. 81, 3741 (1997).

${ }^{4}$ H. Boeve, E. Girgis, J. Schelten, J. De Boeck, and G. Borghs, Appl. Phys. Lett. 76, 1048 (2000).

${ }^{5}$ H. Boeve, R. J. M. van de Veerdonk, B. Dutta, J. De Boeck, J. Moodera, and G. Borghs, J. Appl. Phys. 83, 6700 (1998).

${ }^{6}$ J. S. Moodera, L. R. Kinder, J. Nowak, P. LeClair, and R. Meservey, Appl. Phys. Lett. 69, 708 (1996).

${ }^{7}$ P. K. Wong, J. E. Evetts, and M. G. Blamire, J. Appl. Phys. 83, 6697 (1998).

${ }^{8}$ T. Miyazaki and N. Tezuka, J. Magn. Magn. Mater. 139, 1231 (1995).

${ }^{9}$ S. S. P. Parkin, R. E. Fontana, and A. C. Marley, J. Appl. Phys. 81, 5521 (1997).

${ }^{10}$ P. Rottläunder, H. Kohlstedt, H. A. M. de Gronckel, E. Girgis, J. Schelten, and P. Grünberg, J. Magn. Magn. Mater. 210, 251 (2000).

${ }^{11}$ P. Rottländer, H. Kohlstedt, P. Grünberg, and E. Girgis, J. Appl. Phys. 87, 6067 (2000).

${ }^{12}$ R. Schad, D. Allen, G. Zangari, I. Zana, D. Yang, M. Tondra, and D. Wang, Appl. Phys. Lett. 76, 607 (2000).

${ }^{13}$ N. Garcia, M. Munoz, and Y. W. Zhao, Phys. Rev. Lett. 82, 2923 (1999).

${ }^{14}$ G. Tatara, Y. W. Zhao, M. Munoz, and N. Garcia, Phys. Rev. Lett. 83, 2030 (1999).

${ }^{15}$ N. Garcia, Appl. Phys. Lett. 77, 1351 (2000).

${ }^{16}$ P. M. Levy and S. Zhang, Phys. Rev. Lett. 79, 5110 (1997)

${ }^{17}$ U. Rüdiger, J. Yu, L. Thomas, S. S. P. Parkin, and A. D. Kent, Phys. Rev. B 59, 11914 (1999).

${ }^{18}$ U. Ebels, A. Radulescu, Y. Henry, L. Piraux, and K. Ounadjela, Phys. Rev. Lett. 84, 983 (2000).

${ }^{19}$ Tunneling Phenomena in Solids, edited by E. Burnstein and S. Lundqvist (Plenum, New York, 1969).

${ }^{20}$ B. J. Jönsson-Åkerman, R. Escudero, C. Leighton, S. Kim, I. K. Schuller, and D. A. Rabson, Appl. Phys. Lett. 77, 1870 (2000).

${ }^{21}$ D. A. Rabson, B. J. Jönsson-Åkerman, A. H. Romero, R. Escudero, C. Leighton, S. Kim, and I. K. Schuller, J. Appl. Phys. (submitted).

${ }^{22}$ U. May, K. Samm, H. Kittur, J. O. Hauch, R. Calarco, U. Rüdiger, and G. Güntherodt, Appl. Phys. Lett. (submitted).

${ }^{23}$ C. H. Shang, J. Nowak, R. Jansen, and J. S. Moodera, Phys. Rev. B 58, R2917 (1998).

${ }^{24}$ I. Mertig (private communication).

${ }^{25}$ I. I. Oleinik, E. Y. Tsymbal, and D. G. Pettifor, Phys. Rev. B 62, 3952 (2000).

${ }^{26}$ E. Y. Tsymbal and D. G. Pettifor, Phys. Rev. B 58, 432 (1998).

${ }^{27}$ E. Y. Tsymbal and D. G. Pettifor, J. Appl. Phys. 85, 5801 (1999).

${ }^{28}$ I. Campell and A. Fert, in Ferromagnetic Materials, edited by E. Wohlfarth (North-Holland, Amsterdam, 1982), Vol. 3.

${ }^{29}$ The micromagnetic calculations have been performed using the OOMMF code from the National Institute of Standards and Technology (NIST). Details about this micromagnetic calculation will be published elsewhere. 